\title{
Transient Convective Heat Transfer Flow of a Viscous Dissipative Fluid through a Porous Medium in a Vertical Channel with Radiation
}

\author{
Dr.G.Kathyayani ${ }^{1}$, M. Swapna Priya ${ }^{2}$,D.M.Praveen Babu ${ }^{3}$ \\ Assistant Professor, Department of Applied Mathematics, YogiVemana University,Kadapa ${ }^{l}$ \\ M.Phil Scholar,Department of AppliedMathematics, S.V.University,Tirupati ${ }^{2}$. \\ Ph.D Scholar,Department of Applied Mathematics, YogiVemana University,Kadapa ${ }^{3}$.
}

\begin{abstract}
We discuss the effect of radiation on unsteady MHD free connective flow through a porous medium in a vertical channel. The unsteadiness in the flow is due to the traveling thermal wave imposed on the wall $y=$ $L$. A uniform magnetic field of strength Ho is applied normal to the boundaries. The coupled equations governing the flow and heat transfer have been solved by using a perturbation technique with the aspect ratio as perturbation parameter. The expression for the velocity, the temperature, the shear stress and the rate of heat transfer are derived and are analysed for different variations of the governing parameters $G, R, M, \alpha$ and $\gamma$ Key Words:-Dissipative fluid, Porous medium, Radiation
\end{abstract}

\section{Introduction:-}

The energy crisis has been a topic of great importance in recent years all over the world .This has resulted in an unabated exploration for new ideas and avenues in harnessing various conventional energy sources like tidal waves, wind power and geothermal energy .It is well known that in order to harness maximal geothermal energy one should have complete and precise knowledge of quanta of perturbation needed to initiate convection currents in mineral fluids embedded in the earth's crest enables one to use mineral energy to extract the minerals .For example, in the recovery of hydrocarbons from underground petroleum reservoirs, the use of thermal processes is becoming important to enhance the recovery. Heat can be injected into the reservoir as hot water or steam or heat can be generated inside by burning part of the reservoir crude.

In the theory of flow through porous medium, the role of momentum equations or force balance is occupied by the numerous experimental observations summerised mathematically as the Darcy's law. It is observed that the Darcy's law is applicable as long as the Reynolds number based on average grain(pore) diameter does not exceed a value between 1 and 10.But in general, the speed of specific discharge in the medium need not be always low. As the specific discharge increases, the convective forces get developed and the internal stress generated in the fluid due to its viscous nature produces distortions in the velocity field. Also in the case of highly porous media such as fiber glass, Pappas of dandelion etc., the viscous stress at the surface is able to penetrate into media and produce flow near the surface even in the absence of the pressure gradient. Thus Darcy's law which specifies a linear relationship between the specific discharge and hydraulic gradient is inadequate in describing high speed flows or flows near surfaces which may be either permeable or not. Hence consideration for non-Darcian description for the viscous flow through porous media is warranted. Saffman (6) employing statistical method derived a general governing equations for the flow in a porous medium which takes into account the viscous stress. Later another modification has suggested by Brinkman (1)

$$
o=-\nabla p-\left(\frac{\mu}{k}\right) \bar{v}+\mu \nabla^{2} \bar{v}
$$

in which $\mu \nabla^{2} \bar{v}$ is intended to account for the distortions of the velocity profiles near the boundary. The same equation was derived analytically by Tam (7) to describe the viscous flow at low Reynolds number pasra swam of small particles. By using Darcy's law Yamamoto and Yoshida(8) considered suction and injection flow with convective accelerations through a plane porous wall specifically for flow outside a vortex layer. The generalization of the above study was presented by Yamamoto and Iwamura (9).Chawla and Singh(2) studied oscillatory flow past a porous bed. The steady two-dimensional flow of viscous fluid through a porous medium bounded by porous surface subjected to a constant suction velocity by taking account of free convection currents ( both velocity and temperature fields are constant along $\mathrm{x}$-axis)was studied by Raptis et al., (4).

There is an extensive literature on free convection in porous media ,i.e ,flows through a porous media under gravitational fields that are driven by gradients of fluid density caused by temperature gradient.

Convection fluid flows generated by traveling thermal waves have also received attention due to applications in physical problems. From a physical point of view, the motion induced by traveling thermal 
waves is quite interesting as a purely fluid-dynamical problem and can be used as a possible explanation for the observed four-day retrograde zonal motion of the upper atmosphere of Venus. All the above mentioned studies are based on the hyphothesis that the effect of dissipation is neglected. This is possible in case of ordinary fluid flow like air and water under gravitational force .But this effect is expected to be relevant for fluids with high values of the dynamic viscosity flows. Moreover many have studied the effect of viscous dissipation on the convective flows past an infinite vertical plate and through vertical channels and ducts.

The effect of viscous dissipation on natural convection has been studied for some different cases including the natural convection from horizontal cylinder embedded in a porous media by Fand and Brucker(3).Ravindra(5) has analysed the effect of traveling thermal wave on MHD convective flow of a viscous electrically conducting fluid through a porous medium in a vertical channel.

\section{Formulation Of The Problem:-}

We consider the motion of viscous, incompressible fluid through a porous medium in a vertical channel bounded by flat walls. The thermal buoyancy in the flow field is created by a traveling thermal wave imposed on the boundary wall at $\mathrm{y}=\mathrm{L}$ while the boundary at $\mathrm{y}=-\mathrm{L}$ is maintained at constant temperature $\mathrm{T}_{1}$. The Boussinesq approximation is used so that the density variation will be considered only in the buoyancy force. The viscous and Darcy dissipations are taken into account to the transport of heat by conduction and convection in the energy equation. Also the kinematics viscosity $v$, the thermal conducting $\mathrm{k}$ are treated as constants. We choose a rectangular Cartesian system $0(\mathrm{x}, \mathrm{y})$ with $\mathrm{x}$-axis in the vertical direction and $\mathrm{y}$-axis normal to the walls. The walls of the channel are at

$\mathrm{y}= \pm \mathrm{L}$.

The equations governing the unsteady flow and heat transfer are

\section{Equation of continuity}

$$
\frac{\partial u}{\partial x}+\frac{\partial v}{\partial y}=0
$$

Equation of linear momentum

$$
\begin{gathered}
\rho_{e}\left(\frac{\partial u}{\partial t}+u \frac{\partial u}{\partial x}+v \frac{\partial u}{\partial y}\right)=-\frac{\partial p}{\partial x}+\mu\left(\frac{\partial^{2} u}{\partial x^{2}}+\frac{\partial^{2} u}{\partial y^{2}}\right)-\rho g--\left(\frac{\mu}{k}\right) u \\
\rho_{e}\left(\frac{\partial v}{\partial t}+u \frac{\partial v}{\partial x}+v \frac{\partial v}{\partial y}\right)=-\frac{\partial p}{\partial y}+\mu\left(\frac{\partial^{2} v}{\partial x^{2}}+\frac{\partial^{2} v}{\partial y^{2}}\right)-\left(\frac{\mu}{k}\right) v
\end{gathered}
$$

\section{Equation of Energy:-}

$$
\begin{aligned}
\rho_{e} C_{p}\left(\frac{\partial T}{\partial t}+u \frac{\partial T}{\partial x}+v \frac{\partial T}{\partial y}\right) & =\lambda\left(\frac{\partial^{2} T}{\partial x^{2}}+\frac{\partial^{2} T}{\partial y^{2}}\right)+Q-\frac{\partial q_{r}}{\partial y} \\
& +\mu\left(\left(\frac{\partial u}{\partial y}\right)^{2}+\left(\frac{\partial v}{\partial x}\right)^{2}\right)+\left(\frac{\mu}{\lambda k}\right)\left(u^{2}+v^{2}\right)
\end{aligned}
$$

Equation of state :-

$$
\rho-\rho_{e}=-\beta \rho_{e}\left(T-T_{e}\right)
$$

where $\rho_{e}$ is the density of the fluid in the equilibrium state, are the temperature in the equilibrium state, $(\mathrm{u}, \mathrm{v})$ are the velocity components along $\mathrm{O}(\mathrm{x}, \mathrm{y})$ directions, $\mathrm{p}$ is the pressure, $\mathrm{T}$ is the temperature in the flow region, $\rho$ is the density of the fluid, $\mu$ is the constant coefficient of viscosity, $\mathrm{Cp}$ is the specific heat at constant pressure, $\lambda$ is the coefficient of thermal conductivity, $\mathrm{k}$ is the permeability of the porous medium , $\beta$ is the coefficient of thermal expansion, $\mathrm{Q}$ is the strength of the constant internal heat source and $\mathrm{q}_{\mathrm{r}}$ is the radioactive heat flux.

By Rosseland approximation(3a) the radioactive heat flux is given by

$$
q_{r}=\frac{4 \sigma^{\bullet}}{3 \beta_{r}} \frac{\partial T^{\prime 4}}{\partial y}
$$

and by Taylor's approximation we have 


$$
T^{\prime 4} \cong 4 T_{e}^{3} T-3 T_{e}^{4}
$$

after neglecting higher powers of $\mathrm{T}$.

In the equilibrium state

$$
0=-\frac{\partial p_{e}}{\partial x}-\rho_{e} g
$$

where $p=p_{e}+p_{D}, p_{D}$ being the hydrodynamic pressure.

The flow is maintained by a constant volume flux for which a characteristic velocity is defined as

$$
Q=\frac{1}{2 L} \int_{-L}^{L} u d y
$$

The boundary conditions for the velocity and temperature fields are

$$
\begin{aligned}
& \mathrm{u}=0, \mathrm{v}=0, \mathrm{~T}=\mathrm{T}_{1}, \mathrm{C}=\mathrm{C}_{1} \\
& u=0, v=0, T=T_{2}+\Delta T_{e} \operatorname{Sin}(m x+n t), C=C_{2} \quad \text { on } \mathrm{y}=-\mathrm{L}
\end{aligned}
$$

where $\Delta T_{e}=T_{2}-T_{1}$ and $\operatorname{Sin}(m x+n t)$ is the imposed traveling thermal wave In view of the continuity equation we define the stream function $\psi$ as

$$
\mathrm{u}=-\psi_{\mathrm{y}}, \mathrm{v}=\psi_{\mathrm{x}}
$$

Eliminating pressure $\mathrm{p}$ from equations (2.2)\&(2.3)and using the equations governing the flow in terms of $\psi$ are

$$
\begin{aligned}
& {\left[\left(\nabla^{2} \psi\right)_{t}+\psi_{x}\left(\nabla^{2} \psi\right)_{y}-\psi_{y}\left(\nabla^{2} \psi\right)_{x}\right] }=v \nabla^{4} \psi-\beta g\left(T-T_{0}\right)_{y}--\left(\frac{v}{k}\right) \nabla^{2} \psi \\
& \rho_{e} C_{p}\left(\frac{\partial \theta}{\partial t}+\frac{\partial \psi}{\partial y} \frac{\partial \theta}{\partial x}-\frac{\partial \psi}{\partial x} \frac{\partial \theta}{\partial y}\right)=\lambda \nabla^{2} \theta+Q+\mu\left(\left(\frac{\partial^{2} \psi}{\partial y^{2}}\right)^{2}+\left(\frac{\partial^{2} \psi}{\partial x^{2}}\right)^{2}\right)+ \\
&\left.+\left(\frac{\mu}{k}\right)\left(\left(\frac{\partial \psi}{\partial x}\right)^{2}+\left(\frac{\partial \psi}{\partial y}\right)^{2}\right)\right)+\frac{16 \sigma^{\bullet} T_{e}^{3}}{3 \beta_{R}} \frac{\partial^{2} T}{\partial y^{2}}
\end{aligned}
$$

Introducing the non-dimensional variables in (2 .9)\& (2.10) as

$$
x^{\prime}=m x, y^{\prime}=y / L, t^{\prime}=t v m^{2}, \Psi^{\prime}=\Psi / v, \theta=\frac{T-T_{2}}{\Delta T_{e}}
$$

the governing equations in the non-dimensional form (after dropping the dashes ) are

$$
\delta R\left(\delta\left(\nabla_{1}^{2} \psi\right)_{t}+\frac{\partial\left(\psi, \nabla_{1}^{2} \psi\right)}{\partial(x, y)}\right)=\nabla_{1}^{4} \psi+\left(\frac{G}{R}\right) \theta_{y}-D^{-1} \nabla_{1}^{2} \psi
$$

and the energy equation in the non-dimensional form is

$$
\begin{gathered}
\delta P\left(\delta \frac{\partial \psi}{\partial t}+\frac{\partial \psi}{\partial y} \frac{\partial \theta}{\partial x}-\frac{\partial \psi}{\partial x} \frac{\partial \theta}{\partial y}\right)=\nabla_{1}^{2} \theta+\alpha+\left(\frac{P R^{2} E_{c}}{G}\right)\left(\left(\frac{\partial^{2} \psi}{\partial y^{2}}\right)^{2}+\delta^{2}\left(\frac{\partial^{2} \psi}{\partial x^{2}}\right)^{2}\right)+ \\
\left.+D^{-1}\left(\delta^{2}\left(\frac{\partial \psi}{\partial x}\right)^{2}+\left(\frac{\partial \psi}{\partial y}\right)^{2}\right)\right)+\frac{4}{3 N} \frac{\partial^{2} \theta}{\partial y^{2}}
\end{gathered}
$$

where

$$
\begin{array}{lr}
R=\frac{U L}{v} \quad \text { (Reynolds number), } & G=\frac{\beta g \Delta T_{e} L^{3}}{v^{2}} \text { (Grashof number), } \\
\mathrm{P}=\frac{\mu c_{p}}{k_{1}}(\text { Prandtl number), } & D^{-1}=\frac{L^{2}}{k} \text { (Darcy parameter), } \\
E_{c}=\frac{\beta g L^{3}}{C_{p}} \text { (Eckert number), } & \delta=m L \quad \text { (Aspect ratio), } \\
\gamma=\frac{n}{m^{2}} \text { (non-dimensional thermal wave velocity), } N=\frac{3 \beta_{R}}{4 \sigma^{*} T_{e}^{3}} \quad \text { (Radiation parameter), }
\end{array}
$$




$$
\begin{gathered}
P_{1}=\frac{3 N P}{3 N+4} \quad \alpha_{1}=\frac{3 N \alpha}{3 N+4} \\
\nabla_{1}^{2}=\delta^{2} \frac{\partial^{2}}{\partial x^{2}}+\frac{\partial^{2}}{\partial y^{2}} \quad \begin{array}{c}
\text { The corresponding boundary conditions are } \\
\psi(+!)-\psi(-!)=1
\end{array} \\
\frac{\partial \psi}{\partial x}=0, \quad \frac{\partial \psi}{\partial y}=0 \quad \text { at } y= \pm 1 \\
\theta(x, y)=1 \quad, C=0 \quad \text { on } \quad \mathrm{y}=-1 \\
\theta(x, y)=\operatorname{Sin}(x+\gamma t), C=1 \quad \text { at } \quad y=0 \\
\frac{\partial \theta}{\partial y}=0, \frac{\partial C}{\partial y}=0 \quad
\end{gathered}
$$

The value of $\psi$ on the boundary assumes the constant volumetric flow in consistent with the hypothesis (2.7) .Also the wall temperature varies in the axial direction in accordance with the prescribed arbitrary function $\mathrm{t}$.

\section{Analysis Of The Flow:-}

The main aim of the analysis is to discuss the perturbations created over a combined free and forced convection flow due to traveling thermal wave imposed on the boundaries. The perturbation analysis is carried out by assuming that the aspect ratio $\delta$ to be small.

We adopt the perturbation scheme and write

$$
\begin{aligned}
& \psi(x, y, t)=\psi_{0}(x, y, t)+\delta \psi_{1}(x, y, t)+\delta^{2} \psi_{2}(x, y, t)+. \\
& \dot{\theta}(x, y, t)=\theta_{0}(x, y, t)+\delta \theta_{1}(x, y, t)+\delta^{2} \theta_{2}(x, y, t)+
\end{aligned}
$$

On substituting ( 3.1) in (2.13) - (2.15) and separating the like powers of $\delta$ the equations and respective conditions to the zeroth order are

$$
\begin{aligned}
& \psi_{0, y y y y y}-M_{1}^{2} \psi_{0, y y}=-G\left(\theta_{0, y}+N C_{0, y}\right) \\
& \theta_{o, y y}+\alpha_{1}+\frac{P_{1} E_{c} R^{2}}{G}\left(\psi_{o, y y}\right)^{2}+\frac{P_{1} E_{c}\left(D^{-1}+M^{2}\right)}{G}\left(\psi_{o, y}^{2}\right)=0
\end{aligned}
$$

with

$$
\psi_{0, \mathrm{y}}=0, \psi_{0, \mathrm{x}}=0 \quad \text { at } \mathrm{y}= \pm 1
$$

$\theta_{o}=1 \quad$ on $y=-1$

$\theta_{o}=\operatorname{Sin}(x+\not t)$ on $y=1$

and to the first order are

$$
\begin{aligned}
& \psi_{1, y y y y}-M_{1}^{2} \psi_{1, y y}=-G \theta_{y}+\left(\psi_{0, y} \psi_{0, x y y}-\psi_{0, x} \psi_{0, y y y}\right) \\
& \theta_{1, y y}=\left(\psi_{0, x} \theta_{o, y}-\psi_{0, y} \theta_{o x}\right)+\frac{2 P_{1} E_{c} R^{2}}{G}\left(\psi_{0, y y} \psi_{1, y y}\right)+\frac{2 P_{1} E_{c} D^{-1}}{G}\left(\psi_{0, y} \cdot \psi_{1, y}\right)
\end{aligned}
$$

With $\psi_{1(+1)}-\psi_{1(-1)}=0$

$$
\begin{aligned}
& \psi_{1, y}=0, \psi_{1, \mathrm{x}}=0 \text { at } \mathrm{y}= \pm 1 \\
& \theta_{1}( \pm 1)=0 \quad \text { at } \mathrm{y}= \pm 1
\end{aligned}
$$

Assuming Ec $<<1$ to be small we take the asymptotic expansions as 


$$
\begin{aligned}
& \psi_{0}(x, y, t)=\psi_{00}(x, y, t)+E c \psi_{01}(x, y, t)+ \\
& \psi_{1}(x, y, t)=\psi_{10}(x, y, t)+E c \psi_{11}(x, y, t)+ \\
& \theta_{0}(x, y, t)=\theta_{00}(x, y, t)+\theta_{01}(x, y, t)+. . \\
& \theta_{1}(x, y, t)=\theta_{10}(x, y, t)+\theta_{11}(x, y, t)+\ldots \ldots . .
\end{aligned}
$$

Substituting the expansions(3.10) in equations (3.2)-(3.9)and separating the like powers- of Ec we get the following

$$
\begin{aligned}
& \theta_{00, y y}=-\alpha_{1} \quad, \theta_{00}(-1)=1, \theta_{00}(+1)=\operatorname{Sin} D_{1} \\
& \psi_{00, y y y y}-M_{1}^{2} \psi_{00, y y}=-G \theta_{00, y}, \\
& \psi_{00}(+1)-\psi_{00}(-1)=1, \psi_{00, y}=0, \psi_{00, x}=0 \text { at } y= \pm 1 \\
& \theta_{01, y y}=-\frac{P_{1} R}{G} \psi^{2}{ }_{00, y y}-\frac{P_{1} D^{-1}}{G} \psi_{00, y}^{2} \quad, \quad \theta_{01}( \pm 1)=0 \\
& \psi_{01, y y y y}-M_{1}^{2} \psi_{01, y y}=-G \theta_{01, y} \quad \theta_{10}( \pm 1)=0 \\
& \psi_{01}(+1)-\psi_{01}(-1)=0, \psi_{01, y}=0, \psi_{01, x}=0 \text { at } y= \pm 1 \\
& \theta_{10, y y}=\left(\psi_{00, y} \theta_{00, x}-\psi_{00, x} \theta_{00, y}\right) \quad \theta^{2}
\end{aligned}
$$

$$
\psi_{10, y y y y}-M_{1}^{2} \psi_{10, y y}=-G \theta_{10, y}+\left(\psi_{00, y} \psi_{00, x y y}-\psi_{00, x} \psi_{00, y y y}\right)
$$

$$
\psi_{10}(+1)-\psi_{10}(-1)=0, \psi_{10, y}=0, \psi_{10, x}=0 \text { at } y= \pm 1
$$

$$
\theta_{11, y y}=\left(\psi_{00, y} \theta_{01, x}-\psi_{01, x} \theta_{00, y}+\theta_{00, x} \psi_{01, y}-\theta_{01, y} \psi_{0, x}\right)-\frac{2 P_{1} R^{2}}{G} \psi_{00, y y} \psi_{10, y y}
$$

$$
\psi_{11, y y y y}-M_{1}^{2} \psi_{1, y y}=-G \theta_{1, y}+\left(\psi_{00, y} \psi_{11, x y y}-\psi_{00, x} \psi_{01, y y y}+\psi_{01, y} \psi_{00, x y y}-\psi_{01 . x} \psi_{00, y y y}\right) \text {, }
$$

$$
-\frac{2 P_{1} D^{-1}}{G} \psi_{00, y} \psi_{10, y}, \theta_{1}( \pm 1)=0
$$

$$
\psi_{11}(+1)-\psi_{11}(-1)=0, \psi_{11, y}=0, \psi_{11, x}=0 \text { at } y= \pm 1
$$

\section{Solution Of The Problem:-}

Solving the equations (3.11)- (3.18) subject to the relevant boundary conditions we obtain $\theta_{o o}(y, t)=\left(\frac{\alpha_{1}}{2}\right)\left(1-y^{2}\right)+\left(\frac{\operatorname{Sin}\left(D_{1}\right)-1}{2}\right) y+\left(\frac{\operatorname{Sin}\left(D_{1}\right)+1}{2}\right)$ 


$$
\begin{aligned}
& \psi_{o o}(y, t)=a_{5}\left(\frac{\operatorname{Sh}\left(\beta_{1} y\right)}{\beta_{1}}-y \operatorname{Ch}\left(\beta_{1}\right)\right)+5 a_{3}\left(\frac{y^{5}}{5}-y\right)+14 a_{4}\left(\frac{y^{4}}{4}+\frac{C h\left(\beta_{1} y\right)}{\beta_{1} \operatorname{Sh}\left(\beta_{1}\right)}\right) \\
& \theta_{01}(y, t)=b_{3} y^{4}+b_{4} C h\left(2 \beta_{1} y\right)+b_{11} \operatorname{Sh}\left(2 \beta_{1} y\right)+b_{13} y^{6}+b_{16} y^{8}+b_{17} y^{7}+b_{19} y^{9}+ \\
& \begin{array}{l}
+b_{39}+b_{40} y+b_{41} \operatorname{Sh}\left(\beta_{1} y\right)+b_{42} \operatorname{Ch}\left(\beta_{1} y\right)+b_{43} y^{5} \\
\psi_{01}(y, t)=d_{1}+d_{2} y+\operatorname{PR}^{2}\left(-d_{8} \operatorname{Sh}\left(2 \beta_{1} y\right)-d_{10} y^{10}-d_{15} y^{3} \operatorname{Sh}\left(\left(2 \beta_{1} y\right)\right)+\right.
\end{array} \\
& +P_{1} D^{-1}\left(-d_{21} \operatorname{Sh}\left(2 \beta_{1} y\right)+d_{30} y^{12}+d_{32} y^{8}+d_{36} \operatorname{Ch}\left(\left(2 \beta_{1} y\right)+d_{38} \operatorname{Ch}\left(\beta_{1} y\right)+\right.\right. \\
& \begin{array}{c}
+d_{39} \operatorname{Sh}\left(\left(\beta_{1} y\right)+d_{40} y^{9}+d_{41} y^{11}+d_{42} y^{4}+d_{43} y^{5}\right. \\
\theta_{10}(y, t)=-d_{44}\left(y \operatorname{Ch}\left(\left(\beta_{1} y\right)-\operatorname{Ch}\left(\beta_{1}\right)\right)+d_{45}\left(y^{5}-y\right)+d_{46}\left(\operatorname{Sh}\left(\beta_{1} y\right)-y \operatorname{Sh}\left(\beta_{1}\right)\right)+\right.
\end{array} \\
& +d_{47}\left(\operatorname{Ch}\left(\beta_{1} y\right)-\operatorname{Ch}\left(\beta_{1}\right)\right)-d_{48}\left(y^{2}-1\right)+d_{49}\left(y^{7}-y\right)+d_{50}\left(y^{6}-1\right)-d_{51}\left(y^{3}-y\right) \\
& \theta_{11}=h_{67}+h_{68} \operatorname{Sh}\left(\beta_{1} y\right)+h_{69} C h\left(\beta_{1} y\right)+h_{70} \operatorname{Sh}\left(2 \beta_{1} y\right)+h_{59} C h\left(2 \beta_{1} y\right)+ \\
& +h_{61} y^{9}+h_{62} y^{8}+h_{63} y^{7}+h_{27} y^{6}+h_{28} y^{5}+h_{64} y^{4}+h_{48} y^{3}+h_{71} y
\end{aligned}
$$

where $a_{1}, a_{2}, \ldots \ldots \ldots \ldots . a_{5}, b_{3}, b_{4}, \ldots \ldots \ldots \ldots b_{43}, d_{2}, \ldots \ldots . d_{53}, e_{1}, \ldots \ldots \ldots, e_{36}, h_{1}, \ldots \ldots, h$

$$
i_{1}, \ldots \ldots . . ., i_{78}, l_{1}, \ldots \ldots, l_{50}
$$

are constants given in the appendix.

\section{SHEAR STRESS And NUSSELT NUMBER:-}

The shear stress on the channel walls is given by

$$
\tau=\mu\left(\frac{\partial u}{\partial y}+\frac{\partial v}{\partial x}\right) y= \pm L
$$

which in the non- dimensional form reduces to

$$
\begin{aligned}
\tau=\left(\frac{\frac{\tau U}{a}}{a}\right) & =\left(\psi_{y y}-\delta^{2} \psi_{x x}\right) \\
& =\left[\psi_{00, y y}+E c \psi_{01, y y}+\delta\left(\psi_{10, y y}+E c \psi_{11, y y}+O\left(\delta^{2}\right)\right]_{y= \pm 1}\right.
\end{aligned}
$$

and the corresponding expressions are

$$
\begin{aligned}
& (\tau)_{y=+1}=d_{3}+E c d_{4}+\delta d_{5}+O\left(\delta^{2}\right) \\
& (\tau)_{y=-1}=d_{6}+E c d_{7}+\delta d_{8}+O\left(\delta^{2}\right)
\end{aligned}
$$

The local rate of heat transfer coefficient( Nusselt number $\mathrm{Nu}$ ) on the walls has been calculated using the formula

$$
N u=\frac{1}{\theta_{m}-\theta_{w}}\left(\frac{\partial \theta}{\partial y}\right)_{y= \pm 1}
$$

where

$$
\theta_{m}=0.5 \int_{-1}^{1} \theta d y
$$

and the corresponding expressions are

$$
(N u)_{y=+1}=\frac{\left(m_{1}+E c m_{2}+\delta m_{3}\right)}{\left(m_{4}+E c m_{5}+\delta m_{6}\right)} \quad, \quad(N u)_{y=-1}=\frac{\left(m_{7}+E c m_{8}+\delta m_{9}\right)}{\left(m_{10}+E c m_{5}+\delta m_{6}\right)}
$$

where $m_{1}, m_{2}, \ldots \ldots \ldots \ldots \ldots \ldots \ldots . . . \ldots, m_{10}$ are constants given in the appendix. 


\section{Discussion Of The Results:-}

The aim of this analysis is to discuss the effect of the dissipation on the convective flow and heat transfer of a viscous fluid through a porous medium confined in a vertical channel on whose walls a traveling thermal wave is imposed. Assuming the Eckert number $E_{c}<<1$ the coupled momentum and energy equations have been solved. The velocity and temperature distributions are analysed for different sets of the governing parameters $\mathrm{G}, \mathrm{R}, \mathrm{D}^{-1}, \mathrm{~N}, \alpha$ and $\lambda$ and their profiles are plotted in fig $1-10$. In the following discussion we use the notation that the channel walls are heated or cooled according as $\mathrm{G}>0$ or $\mathrm{G}<0$. We set $\delta=0.01$. The actual axial flow is in the vertically downward direction and hence $\mathrm{u}>0$ represent reversal flow. Fig. 1- 3 represent in $\mathrm{G}, \mathrm{R}, \mathrm{D}^{-1}, \mathrm{~N}, \alpha$ and $\lambda$. For $\mathrm{G} \geq 10^{3}$, in fig-1, we find that lesser the permeability of the porous permeability larger $|\mathrm{u}|$ in the flow region. From fig. 2 we find that $|\mathrm{u}|$ reduces with increase in $\gamma$ and the region of reversed flow shrinks in its size with increase in $\gamma$. An increase in the phase $x+\gamma t$ of the boundary temperature reduces $\mathrm{u}$ in the left half and in the right half it redness with $x+\gamma t \leq \pi / 2$ and enhances with higher $x+\gamma t \geq \pi$ [fig 3].

The non-conformity in the boundary temperature gives rise to a secondary velocity (v) in a direction normal to the boundary. The variation of $\mathrm{v}$ with $\mathrm{D}^{-1}$ reveals that lesser the permeability of the porous medium lesser $|\mathrm{v}|$ except in the region $(-0.4,0)$ in which $|\mathrm{v}|$ depreciates and for further lowering of the permeability larger $|\mathrm{v}|$ in the left half and smaller $|\mathrm{v}|$ in the right half of the changed [fig 4]. An increase in $\alpha$ leads to an enhancement in $|\mathrm{v}|$ [fig 5]. The variation of $\mathrm{v}$ with Ec shows that as increase in Ec enhance $|\mathrm{v}|$ in the left half and depreciates $|\mathrm{v}|$ in the right half of the charged (fig-6). From fig-7,8 we find that for radiation parameter $\mathrm{N} \leq 10$ the secondary velocity in the left half is towards the mid region and is towards boundary in the right half. For higher $\mathrm{N} \geq 10, \mathrm{v}$ is towards the boundary. Also $|\mathrm{v}|$ experiences an enhancement with increase in $\mathrm{N}$ in the entire flow region [fig 7].

The temperature distribution $(\theta)$ for different $\mathrm{D}^{-1}, \mathrm{G}, \mathrm{N}, \alpha, \mathrm{Ec}, x+\gamma t$ is depicted in figs $(8-10)$. It is found that $\theta$ is always positive except in a narrow region and is maximum in the mid-region adjacent to $\eta=+1$ [fig 8] represents the temperature with G. It is found that the temperature gradually rises from its value ' $\mathrm{O}$ ' on $\eta$ $=-1$ attains maximum at $\eta=0$ and then falls to its prescribed value $(-1)$ at $\eta=+1$. The temperature reduces with increase in $|\mathrm{G}|(\$ 0)$. . An increase in $\alpha \leq 4$ reduces and its enhances with $\alpha \geq 6$ (fig 9). An increase in the radiation parameter $\mathrm{N} \leq 1.0$ reduces $\theta$ in the flow region and for further $\mathrm{N} \geq 10$, it enhances in the entire region [fig 10].

The shear stress $(\tau)$ at $\eta \pm 1$ have been evaluated for different value of $\mathrm{G}, \mathrm{D}^{-1}, \alpha, \gamma, \mathrm{N}$ and $x+\gamma t$ and are shown in tables (1-2). It is found that $|(\tau)|$ experiences an enhancement with increase in $|G|(\$ 0)$. The variation of $(\tau)$ with $\mathrm{D}^{-1}$ shows that lesser that the permeability of the porous medium larger $|\tau|$ and for further lowering of permeability smaller $|\tau|$ and at $\eta=-1$ larger $|\tau|$. An increase in the enhances the magnitude of $\tau$ at both the walls. Also it enhances with phase velocity $\gamma$ [Table 1 and 2]. The Nusselt number $(\mathrm{Nu})$ is shown in table $(3-4)$ for different parametric values. It is found that the rate of heat transfer depreciate with $|\mathrm{G}|(\$ 0)$. Also lesser the permeability of medium or higher the strength of heat sources larger the rate of heat transfer at $\eta= \pm 1$. Also it enhances with increasing in $\gamma$ at both the walls [table 3,4$]$.

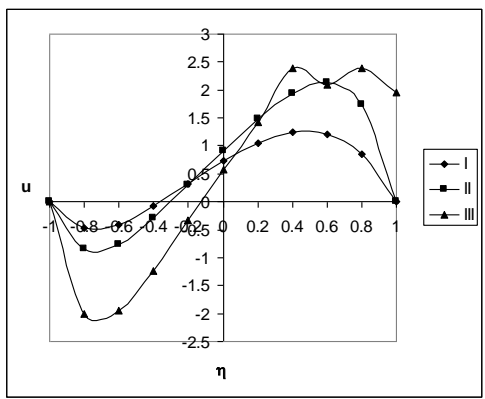

Fig. 1 : variation of $\mathrm{u}$ with $\mathrm{D}^{-1}$

$\begin{array}{llll}\mathrm{D}^{1} & \mathrm{I} & \mathrm{II} & \mathrm{III} \\ & 10^{2} & 2 \times 10^{2} & 3 \times 10^{2}\end{array}$

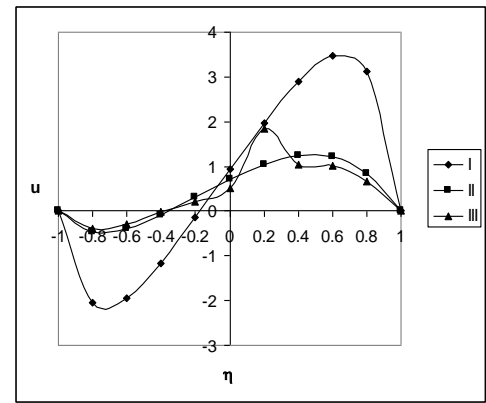

Fig. 2 : variation of $\mathrm{u}$ with $\gamma$

I II III

$\begin{array}{llll}\gamma & 2 & 5 & 10\end{array}$ 


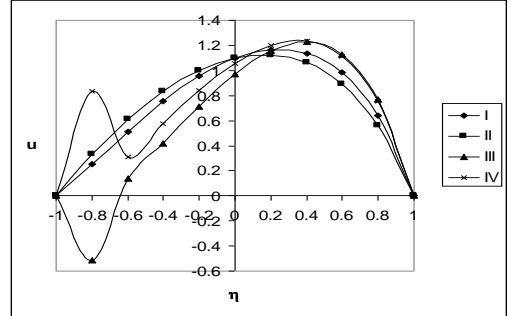

Fig. 3 : variation of $\mathrm{u}$ with $x+\gamma t$

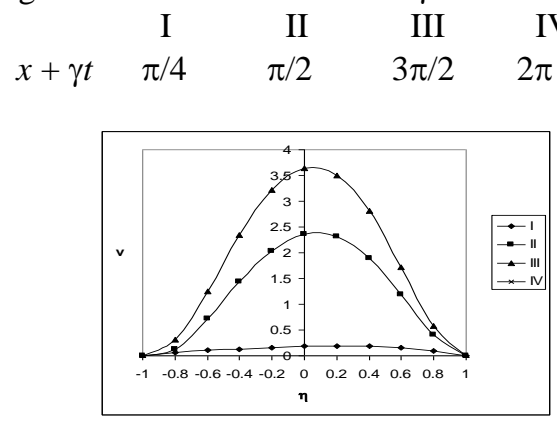

Fig. 5 : Variation of $\mathrm{v}$ with $\alpha$

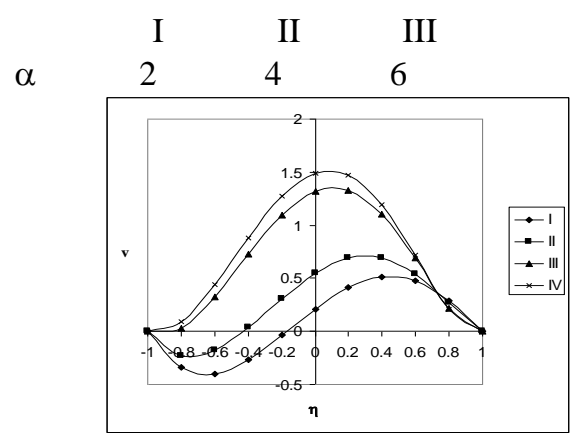

Fig. 7 : variation of $\mathrm{v}$ with $\mathrm{N}$

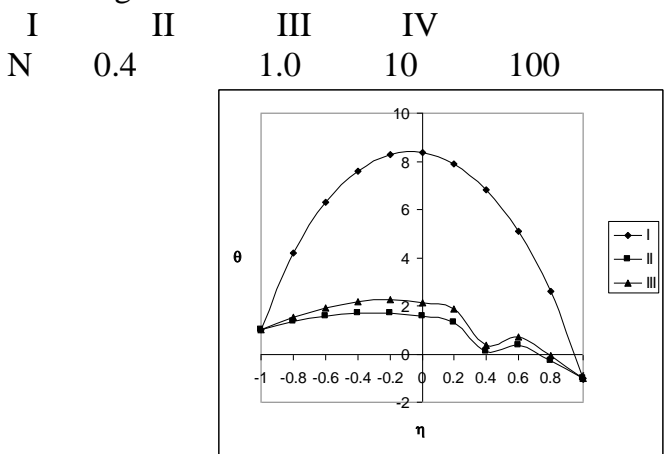

Fig. 9 : Variation of $\theta$ with $\alpha$

$$
\begin{array}{llllll} 
& \text { I } & & \text { II } & \multicolumn{2}{c}{\text { III }} \\
\alpha & & 2 & & 4 &
\end{array}
$$

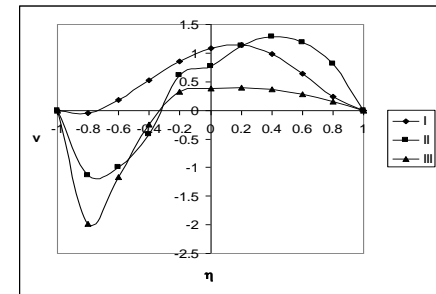

Fig. 4 : variation of $\mathrm{v}$ with $\mathrm{D}^{-1}$ I II III

$\mathrm{D}^{1} \quad 10^{2} \quad 2 \times 10^{2} \quad 3 \times 10^{2}$

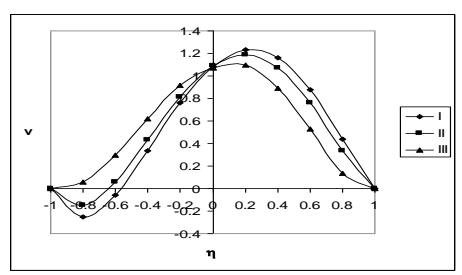

Fig. 6 : variation of $v$ with $E_{c}$

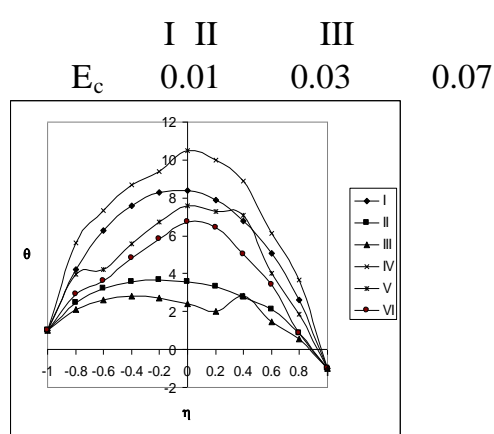

Fig. 8 : Variation of $\theta$ with $\mathrm{G}$

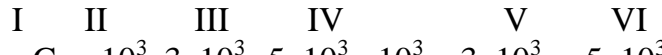
G $\quad 10^{3} \quad 3 \times 10^{3} \quad 5 \times 10^{3}-10^{3} \quad-3 \times 10^{3} \quad-5 \times 10^{3}$

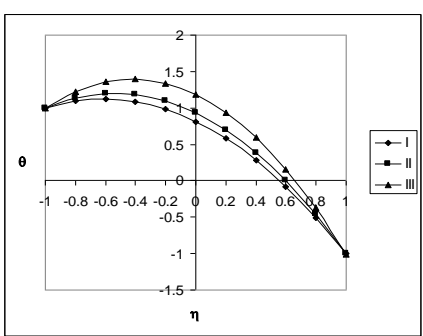

Fig. 10 : variation of $\theta$ with $\mathrm{E}_{\mathrm{c}}$

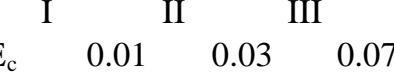

Table -1

Sheer Stress $(\tau)$ at $\mathbf{y}=+1$

\begin{tabular}{|c|c|c|c|c|c|c|}
\hline $\mathrm{G}$ & $\mathrm{I}$ & $\mathrm{II}$ & $\mathrm{III}$ & $\mathrm{IV}$ & $\mathrm{V}$ & VI \\
\hline $10^{3}$ & -3.51284 & -3.765897 & -3.487655 & -3.734573 & -4.134573 & -6.591499 \\
\hline $3 \times 10^{3}$ & -5.251933 & -6.75838 & -6.113057 & -6.844299 & -19.99602 & -20.99602 \\
\hline$-10^{3}$ & -0.041984 & -0.780955 & -1.061721 & -7.51211 & 7.887514 & 8.187514 \\
\hline$-3 \times 10^{3}$ & 3.769490 & 2.203987 & 1.164749 & 2.105787 & -73.02007 & -7.404007 \\
\hline $\mathrm{D}^{-1}$ & $10^{2}$ & $3 \times 10^{2}$ & $5 \times 10^{2}$ & $10^{2}$ & $10^{2}$ & $10^{2}$ \\
\hline$\alpha$ & 2 & 2 & 2 & 4 & 6 & 8 \\
\hline
\end{tabular}


Table - 2

Sheer Stress $(\tau)$ at $\mathbf{y}=-1$

\begin{tabular}{|c|c|c|c|c|c|c|}
\hline $\mathrm{G}$ & $\mathrm{I}$ & $\mathrm{II}$ & $\mathrm{III}$ & $\mathrm{IV}$ & $\mathrm{V}$ & $\mathrm{VI}$ \\
\hline $10^{3}$ & 0.2676 & 1.721 & 5.405 & 1.784 & 2.784 & 3.07 \\
\hline $3 \times 10^{3}$ & -3.206 & -4.249 & 0.1538 & -4.436 & -6.6156 & -0.6156 \\
\hline$-10^{3}$ & 7.214 & 7.691 & 10.26 & 7.751 & 7.95 & 7.45 \\
\hline$-3 \times 10^{3}$ & 14.84 & 13.66 & 14.71 & 13.46 & 16.56 & 23.56 \\
\hline $\mathrm{D}^{-1}$ & $10^{2}$ & $3 \times 10^{2}$ & $5 \times 10^{2}$ & $10^{2}$ & $10^{2}$ & $10^{2}$ \\
\hline$\alpha$ & 2 & 2 & 2 & 4 & 6 & 8 \\
\hline$\gamma$ & 2 & 2 & 2 & 2 & 2 & 10 \\
\hline
\end{tabular}

Table -3

Nusselt Number $(\mathrm{Nu})$ at $\mathrm{y}=+1$

\begin{tabular}{|c|c|c|c|c|c|c|}
\hline $\mathrm{G}$ & $\mathrm{I}$ & $\mathrm{II}$ & $\mathrm{III}$ & $\mathrm{IV}$ & $\mathrm{V}$ & $\mathrm{VI}$ \\
\hline $10^{3}$ & -0.872153 & -1.346310 & -2.743751 & -1.346306 & -1.346310 & -2.905352 \\
\hline $3 \times 10^{3}$ & -0.860067 & -1.343156 & -2.743631 & -1.343142 & -2.587613 & -2.587613 \\
\hline$-10^{3}$ & -0.871738 & -1.345917 & -2.743431 & -1.345922 & -2.909039 & -2.909039 \\
\hline$-3 \times 10^{3}$ & -0.858810 & -1.341975 & -2.742672 & -1.341988 & -2.588409 & -2.588409 \\
\hline $\mathrm{D}^{-1}$ & $10^{2}$ & $3 \times 10^{2}$ & $5 \times 10^{2}$ & $10^{2}$ & $10^{2}$ & $10^{2}$ \\
\hline$\alpha$ & 2 & 2 & 2 & 4 & 6 & 2 \\
\hline$\gamma$ & 2 & 2 & 2 & 2 & 2 & 10 \\
\hline
\end{tabular}

Table -4

Nusselt Number $(\mathrm{Nu})$ at $\mathrm{y}=-1$

\begin{tabular}{|c|c|c|c|c|c|c|}
\hline $\mathrm{G}$ & $\mathrm{I}$ & $\mathrm{II}$ & $\mathrm{III}$ & $\mathrm{IV}$ & $\mathrm{V}$ & $\mathrm{VI}$ \\
\hline $10^{3}$ & -0.37404 & -1.575566 & 9.839230 & -1.575577 & -1.575566 & 3.587615 \\
\hline $3 \times 10^{3}$ & -0.016277 & -1.563764 & 9.834631 & -1.563798 & 25.622230 & 26.622230 \\
\hline$-10^{3}$ & -0.036516 & -1.573224 & 9.844671 & -1.573213 & 3.557781 & 4.557781 \\
\hline$-3 \times 10^{3}$ & -0.013641 & -1.556769 & 9.850973 & -1.556736 & 24.423540 & 26.423540 \\
\hline $\mathrm{D}^{-1}$ & 20 & 30 & 50 & $10^{2}$ & $10^{2}$ & $10^{2}$ \\
\hline$\alpha$ & 2 & 2 & 2 & 4 & 6 & 2 \\
\hline$\gamma$ & 2 & 2 & 2 & 2 & 2 & 10 \\
\hline
\end{tabular}

\section{References:-}

[1]. Brinkman,H.L :A calculation of the viscous force exerted by a flowing on a dense swam of particles.,Appl.Sci.Res,p.82,(1948)

[2]. Chawla,S.S and Singh,S : Oscillatory flow past a porous bed,Acta Mech.,V.34,pp.205-213,(1979)

[3]. Fand,R.M and Brucker,J : Int.J.Heat and Mass Transfer,pp.723-732,(1996)

[4]. Raptis,A,A,Paredikis,C and Zivanidis,G : J.Phys.D :Appl.Phys,V.14,pp.99-102,(1981)

[5]. Ravindra,M: Mhd convection flow through porous medium with non-uniform wall temperature.,Ph.D thesis, S.K.University, Anantapur, India,(1994)

[6]. Saffman,P,G : On the boundary conditions at the force surface of porous medium, Sud..Appl.Maths.,V.2,p.93,(1971)

[7]. Tam,C.K.W : The drag on a cloud of swam of particle in a low Reynolds number flow,J.Fluid Mech.,V.51,p.273,(1969)

[8]. Yamamoto,K and Iwamura,N : J.Engg.Math.,pp.1041-1054,(1976)

[9]. Yamamoto,K and Yoshida,Z : Flow through a porous wall with convective acceleration.,J.Phys,Soc.,Japan,V.37,No.3,pp.774$779,(1974)$ 